\title{
Role of minimally invasive surgery in early ovarian cancer
}

\author{
Chul Kwon Lim, Dae Yeon Kim, Angela Cho, Ji-Yeong Choi, Jeong-Yeol Park, Yong-Man Kim \\ Department of Obstetrics and Gynecology, University of Ulsan College of Medicine, Asan Medical Center, Seoul, Korea \\ Contributions: (I) Conception and design: CK Lim, DY Kim; (II) Administrative support: CK Lim, DY Kim; (III) Provision of study materials or \\ patients: CK Lim, DY Kim; (IV) Collection and assembly of data: JY Park, YM Kim; (V) Data analysis and interpretation: A Cho, JY Choi; (VI) \\ Manuscript writing: All authors; (VII) Final approval of manuscript: All authors. \\ Correspondence to: Dae Yeon Kim. Department of Obstetrics and Gynecology, University of Ulsan College of Medicine, Asan Medical Center, Seoul, \\ Korea. Email: kdyogt@gmail.com.
}

\begin{abstract}
Despite having revolutionized the management of multiple types of gynecologic cancers laparoscopy and robotic surgery have had limited utility in ovarian cancer until recently. The development in medical technology allows surgeons to perform minimally invasive surgery (MIS) not only in early ovarian cancer, but also in advanced ovarian cancer. Thus far, most prospective studies showed feasible results of MIS in ovarian cancer. Even with many proven advantages of the MIS, there is no concrete evidence of the disparity in survival rate between laparoscopic, robotic surgery and laparotomy surgery. We reviewed the results of MIS in ovarian cancer thus far and suggest how the gynecologists can apply MIS in ovarian cancer in the future. Until the further prospective studies show solid evidence of safety in the MIS in ovarian cancer, comprehensive discussion about the benefits and risk with the patient and the level of surgical skill of the gynecologist should be considered in determining the type of surgery.
\end{abstract}

Keywords: Minimally invasive surgical procedures; minimally invasive surgery (MIS); ovarian cancer; laparotomy; laparoscopy; robotic surgery; surgical staging

Submitted Jan 30, 2020. Accepted for publication Jul 15, 2020.

doi: $10.21037 / g s-2019$-ursoc-07

View this article at: http://dx.doi.org/10.21037/gs-2019-ursoc-07

\section{Introduction}

Ovarian cancer is a common type of gynecological cancer, and has a high rate of mortality and recurrence (1). Because ovarian cancer does not have typical symptoms and is located deep within the pelvic cavity, its early diagnosis is particularly difficult; as a result, most cases of ovarian cancer are diagnosed in the advanced stages, which leads to a low 5 -year survival rate and an overall poor prognosis (2).

The International Federation of Gynecology and Obstetrics states that the following factors are closely related to the prognosis of ovarian cancers: histological grade, stage of cancer at diagnosis, and the residual volume of cancer after surgery (3). For patients with stage I cancer, the 5 -year survival rate approaches $90 \%(4,5)$. In most patients with stage III ovarian cancer, the 5 -year survival rate is $46 \%(6-8)$.

Thus, accurate cancer staging is important for prognosis prediction, and staging information has a critical role in adjuvant therapy as well.

Traditionally, ovarian cancer is primarily managed by comprehensive surgical staging through laparotomy such as bilateral salpingo-oophorectomy, total abdominal hysterectomy, omentectomy, aortic and pelvic lymphadenectomy, peritoneal biopsies, and peritoneal washing.

Owing to the technological advancements in instrumentation and an increase in laparoscopic surgical expertise in recent years, minimally invasive surgical have been increasingly used in the management of endometrial cancer and cervical cancer.

Laparoscopic surgery in early-stage ovarian cancer was first described in the mid-1990s (9).

Since that time, several retrospective and case studies have assessed the safety, feasibility, and the potential benefits of laparoscopic surgical staging for ovarian cancers (10-14). 
Laparoscopy provides multiple advantages with better clinical outcomes in terms of less postoperative pain, less blood loss, shorter hospital day, and faster onset chemotherapy than laparotomy (15).

Conversely, laparoscopy has disadvantages such as the inability for lymph node palpation, rupture of the ovarian capsule, and the risk of trocar site metastasis (16).

Robot-assisted surgery also has advantages such as shorter hospital day and less blood loss, but entails higher medical cost. Moreover, robot-assisted surgery has yet to demonstrate a significant clinical benefit over other surgical modalities through randomized controlled trials.

Therefore, it is unclear whether minimally invasive surgery is safe and feasible with early ovarian cancer.

In this review, we compare the surgical and oncological outcomes between laparoscopy, robotic surgery, and laparotomy for the treatment of early ovarian cancer.

\section{Laparoscopic staging procedures}

In the conventional 4 port system, patients are positioned in the Trendelenburg position and a transvaginal uterine manipulator is used. After inserting the first trocar into the umbilical area, $\mathrm{CO}_{2}$ gas $(10-12 \mathrm{mmHg})$ is used to create pneumoperitoneum. Three ancillary trocars-a 5-mm trocar (suprapubic area), a 10-mm trocar (left lower quadrant), and a 5 -mm trocar (right lower quadrant) are inserted under direct vision.

The procedures are led by an assessment of the peritoneal surface and the abdominal and pelvic organs, followed by pelvic washing for cytologic examination. By utilizing an endobag through the $10-\mathrm{mm}$ port in the left lower quadrant, ovarian tumors with intact capsules are removed. After confirming the malignancy using frozen sections, staging surgery including bilateral pelvic and para-aortic lymph node dissection, total laparoscopic hysterectomy with bilateral salpingo-oophorectomy, infracolic omentectomy, and appendectomy are performed. All suspicious areas were biopsied. After hemostasis, the drainage bag was connected through the lateral port.

Recently, with the improvement of instrumentation, a single port or two-port system could be used for staging without compromising surgical qualities.

\section{Robotic staging procedures}

Three camera ports are used in laparoscopic surgery: one is set at the umbilicus, another at $8-10 \mathrm{~cm}$ caudal to the scope on the midline, and the other at $8-10 \mathrm{~cm}$ laterocaudal to the scope on the left side. The fourth port for the assistant is set at $8-10 \mathrm{~cm}$ laterocaudal to the scope on the right side of the patient. The procedures are similar as those for laparoscopic staging.

\section{Laparotomic staging procedures}

Laparotomic staging procedures are similar as those for laparoscopic staging, except for a midline abdominal incision from the superior umbilicus to the symphysis pubis.

\section{Feasibility and efficacy of laparoscopic surgery}

Laparoscopic surgery for ovarian cancer is beneficial due to its minimally invasive nature that allows for reduced blood loss, small wound site, less postoperative pain, and short hospital stay (17-19).

Seven studies have addressed the feasibility, safety was analyzed, the safety and efficacy of laparotomy versus laparoscopy in early-stage ovarian cancer. Those studies were analyzed and shown in Table 1 .

The studies showed that compared with laparotomy, laparoscopic surgery showed significant associations with less blood loss and shorter hospital stays. Most importantly, laparoscopic patients started adjuvant chemotherapy earlier than laparotomy patients.

In our center, Park and colleagues carried out a retrospective, case-controlled comparison of laparoscopy and laparotomy for cancer staging in 52 patients with earlystage cancer in the ovary or the fallopian tube. Laparoscopy and laparotomy showed comparable results in terms of the adequacy and accuracy of surgical staging, while laparoscopy was associated with more favorable outcomes. Moreover, the interval to chemotherapy was shorter $(\mathrm{P}<0.05)$ in those staged by laparoscopy $(12.8 \pm 4.9$ days $)$ than in those staged by laparotomy (17.6 \pm 8.3 days) (23).

Lee et al. retrospectively compared the oncological and surgical outcomes of laparotomy and laparoscopic surgery in 113 patients with early-stage ovarian cancer, and reported that the laparoscopy group had a significantly longer mean operation time than the laparotomy group (227.6 vs. 184.6 minutes, $\mathrm{P}=0.016$ ). The laparoscopy group also had shorter postoperative hospital stay as well as less amount of intraoperative blood loss. Also, the laparoscopy group had a notably lower rate of complications than did the laparotomy group (7.7\% vs. $23.0 \%$ ) (18). Conversely, the two groups did not show significant differences in the 


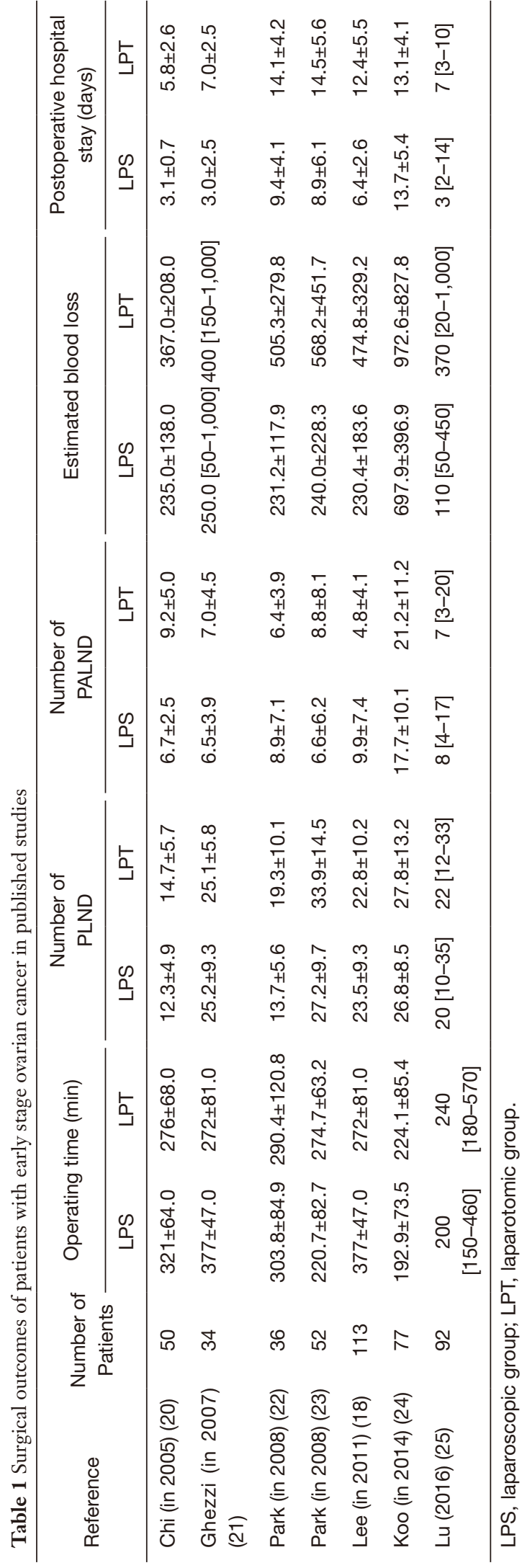

risks of intraoperative mass rupture and port-site metastasis.

Recent studies on early-stage ovarian cancer did not focus on reporting the long-term follow-up data on survival and recurrence. Those studies were analyzed and shown in Table 2. The studies showed that there was no significant difference in the recurrence rate.

Lee et al. reported that eight patients in the laparotomy group showed recurrence whereas patients in the laparoscopic group did not, which may be due to the relatively short follow-up period in the laparoscopic group (18).

\section{Possible risks of laparoscopic approach}

However, laparoscopic surgery still had several unsolved risks.

The first one is difficulty in tumor manipulation, especially in a large and adhesive tumor. Tumor diameter was significantly larger in the laparotomy group. Lee et al. reported that the mean tumor size was larger in the laparotomy group than the laparoscopy group $(14.0 \pm 8.3$ vs. $9.1 \pm 5.0 \mathrm{~cm}, \mathrm{P}=0.01)(18)$. It means that the surgeon can select the surgical method according to the size of the ovary before surgery, which can lead to selection bias.

The second risk is associated with the risk of ovarian tumor rupture during surgery, and many of the reports showed a higher upstaging rate with laparoscopy than with laparotomy $(19,21,26,27)$. Intraoperative tumor rupture results in immediate upstaging and may lead to the peritoneal seeding of cancer cells that may necessitate adjuvant chemotherapy and worsening of prognosis (28).

The third disadvantage is the risk of port-site metastasis. The incidence of port site metastases in invasive ovarian cancer was shown to be up to $16 \%$, whereas Zivanovic et al. reported a recurrence incidence of $1.96 \%$ at the port site alone (29). Accordingly, other recent reports suggested that port-site metastasis in patients whose ovarian cancers are staged by laparoscopy may be rare $(0-2 \%)(30,31)$.

However, a prospective study is needed to better comprehend the actual risk and the prognostic role of laparoscopic staging in ovarian cancer.

\section{Robotic surgery approaches}

Robotic surgery is quickly gaining ground in the field of minimally invasive surgery by overcoming historic barriers of laparoscopy and laparotomy.

Robotic surgery offers three-dimensional (3-D) visualization, tremor-free movements, and improves the 
Table 2 Survival outcomes of patients with early stage ovarian cancer in published studies

\begin{tabular}{|c|c|c|c|c|c|}
\hline Reference & Number of Patients & \multicolumn{2}{|c|}{ Mean follow up (month) } & \multicolumn{2}{|c|}{ Recurrences } \\
\hline Ghezzi (in 2007) (21) & 34 & $16[4-34]$ & 60 [30-108] & 0 & 4 \\
\hline Park (in 2008) (22) & 36 & $19[5-56]$ & $14[5-61]$ & 2 & 0 \\
\hline Park (in 2008) (23) & 52 & 17 [2-40] & $23[1-44]$ & 0 & 0 \\
\hline Koo (in 2014) (24) & 77 & $31.7 \pm 20.7$ & $31.1 \pm 19.1$ & 2 & 2 \\
\hline Qi Lu (in 2016) (25) & 92 & $82[16-152]$ & 82 [16-152] & 5 & 6 \\
\hline
\end{tabular}

LPS, laparoscopic group; LPT, laparotomic group.

dexterity of instrument movement.

As reported, compared to traditional laparotomy and laparoscopy, robotic surgery is safe and feasible for treating cervical cancer with less bleeding and complications and short recovery time (32). Nevertheless, the clinical efficacy of robotic surgery in ovarian cancer has only been reported in a few small-sized retrospective studies.

Feuer $e t a l$. studied the efficacy and feasibility of robotassisted surgery for epithelial ovarian cancer in comparison with laparotomy (33) and showed that while robot-assisted surgeries required significantly longer operative time (138.6 vs. 95.2 minutes), robot-assisted surgeries had less amount of estimated blood loss ( 94.9 vs. $385.4 \mathrm{~mL}$ ) and shorter hospital stay (2.3 vs. 6.2 days).

In another small study, Magrina et al. showed similar results and that robot-assisted group had a significantly higher progression-free survival rate than did the laparotomy group (34).

Several key issues need further research, such as tumor rupture, port-site metastasis, and growth of tumors due to pneumoperitoneum.

\section{Discussion}

Minimally invasive surgery (MIS) has become the trend in surgical techniques in the gynecologic oncology field. Despite existing research on the beneficial role of MIS in the treatment of early-stage cancers in the endometrium and cervix, our current understanding on the role of MIS in early-stage ovarian cancer is lacking. In ovarian cancer, in addition to the treatment of early disease and staging procedures, patients with advanced diseases following neoadjuvant chemotherapy and selected patients with recurrent tumors have become candidates for a minimally invasive approach.

This study aimed to explore the effectiveness of MIS for the treatment of early-stage ovarian cancer. The review showed that confirmed the favorable prognostic outcomes of MIS for reducing the lengths of hospital stays and the less blood loss rates in patients with apparent early-stage ovarian cancer. The number of retrieved lymph nodes and mean operating time were comparable between MIS and laparotomy, while the MIS was associated with a significantly lower incidence of complications.

However, these studies have included a control group between the laparoscopic and laparotomy procedures and limited follow-up periods. Therefore, the role of MIS in patients with early ovarian cancer remains controversial.

The most common controversy on MIS in the treatment of ovarian cancer is the accuracy of complete surgical staging. Among patients with early-stage ovarian cancer, those who have undergone optimal debulking surgery have a lower risk of recurrence than do those who have not. It has been argued that MIS does not allow for a thorough inspection of the pelvis, mesentery, and peritoneum leading to incomplete surgery. In the present review, we did not find significant differences between the laparoscopic group and the laparotomy groups in terms of the number of retrieved lymph nodes. This might be related to the surgeon's experience and competence.

The other controversial issue is the rate of ovarian tumor rupture during surgery. MIS carries the risk of port-site metastasis and spillage, the effects on patient outcome, and the possibility of incomplete tumor staging. Intraoperative ovarian capsular ruptures lead to worsened disease prognosis. Romagnolo et al. showed that tumor rupture or spills are more common in laparoscopic surgery than in laparotomic surgery (34.6\% vs. 6.6\%) (35). However, 
some studies have reported that the risk of tumor rupture is similar between laparoscopic and laparotomic surgery. Park et al. reported that laparotomy and laparoscopy showed similar incidence of tumor rupture in patients with ovarian cancer $(10.5 \%$ vs. $12.1 \% ; \mathrm{P}=1.000)$ (23).

Also of note is that the pattern of disease spread is different in ovarian cancer than in endometrial and cervical cancer. Several studies have reported that laparoscopic surgery may result in the dissemination of ovarian cancer or the aggravation of transperitoneal dissemination. Moreover, tumor cell growth may be promoted by $\mathrm{CO}_{2}$-induced hypercapnia and pneumoperitoneum (36). Also, tumor dissemination and port-site metastasis may be prompted by aerosolization of tumor cells and the chimney effect, a phenomenon in which tumor cells are increased at the port sites due to leakage $(37,38)$.

Nevertheless, most studies so far have demonstrated that surgical staging of early-stage ovarian cancer through laparoscopy is safe.

Accordingly, Lee et al. reported that there were no metastasis at the incision site or the port site (18), which was likely due to their effort in reducing the risk of port-site metastasis by removing intact specimens by a Lap bag and closing the trocar sites by using anatomical layers.

The clinical significance of port site metastasis and intraoperative tumor rupture remains uncertain. Largesized randomized controlled trials are needed to assess the prognostic role of intraoperative tumor rupture in patients with early stage ovarian cancer.

Another important issue is the radical en bloc peritonectomy contributes to optimal cytoreduction affecting prognosis in early ovarian cancer. The aim of radical en bloc peritonectomy is to remove no visible microscopic tumor, as complete debulking is the most important prognostic factor in patients with ovarian cancer. However, radical en bloc peritonectomy is effective in laparotomy and is easier procedure than MIS. Radical en bloc peritonectomy by MIS have different outcomes depending on the level of each surgeon. Also, no studies have described an MIS technique to safely perform radical en bloc peritonectomy.

The last controversy is the higher recurrence rate in laparoscopy. According to the LACC trial, minimally invasive surgery was associated with a lower rate of diseasefree survival in cervical cancer (39). Almost all published data have been from MIS surgeon, who wishes to prove their new technique is feasible, not from the open surgeon.

Also, laparotomy control was older than the MIS case, which means longer follow up and the level of medical practice was low and has lower survival in the laparotomy group. Thus, if the survival was similar in both groups, the MIS could be worse.

We have recently begun a prospective randomized trial comparing laparotomy and laparoscopy (KGOG 3050 trial, Figure 1).

The goal of this study is to compare the surgical and survival outcomes in patients with early-stage epithelial ovarian cancer according to surgical method (laparoscopic/ robotic and open staging surgeries).

However, surgical comparative studies depend on the level of each operator, so there are limits to the application of the findings to all surgeons.

\section{Conclusions}

Laparoscopy and robotic surgery allow optimal visualization of the surgical field as well as better magnification, thereby enabling the detection of implants on the peritoneum surfaces, diaphragm, and the liver. Furthermore, MIS appears to have better surgical outcomes in less blood loss, shorter hospital stay and earlier recovery compared with conventional laparotomy in the treatment of apparent earlystage ovarian tumors.

Several issues such as tumor rupture, port site metastasis, and the enhancement of tumor cell growth due to pneumoperitoneum should be clarified in future studies. Also, there are remaining concerns in terms of the rates of survival and recurrence in patients staged by MIS, as most of the retrospective literature reports survival rates of approximately $90 \%$ at follow-up, which are similar to that observed in patients staged by laparotomy $(14,19)$. But these studies are not long-term data on recurrence and survival. Furthermore, there is still a wide regional variation in the laparoscopic skills and competence of gynecologicaloncology surgeons.

A short and small study cannot easily validate the benefits of MIS for ovarian cancer. There were no reports of a randomized controlled study of MIS for ovarian cancer. Therefore, large prospective studies, like in KGOG, are required to confirm these findings and to develop selection criteria for MIS of ovarian cancer. Until then, comprehensive discussion about the risks and benefits with the patient should be required and the level of surgical skill of the gynecologist should be considered in determining the type of surgery.

Considering these limitations, successful MIS staging 

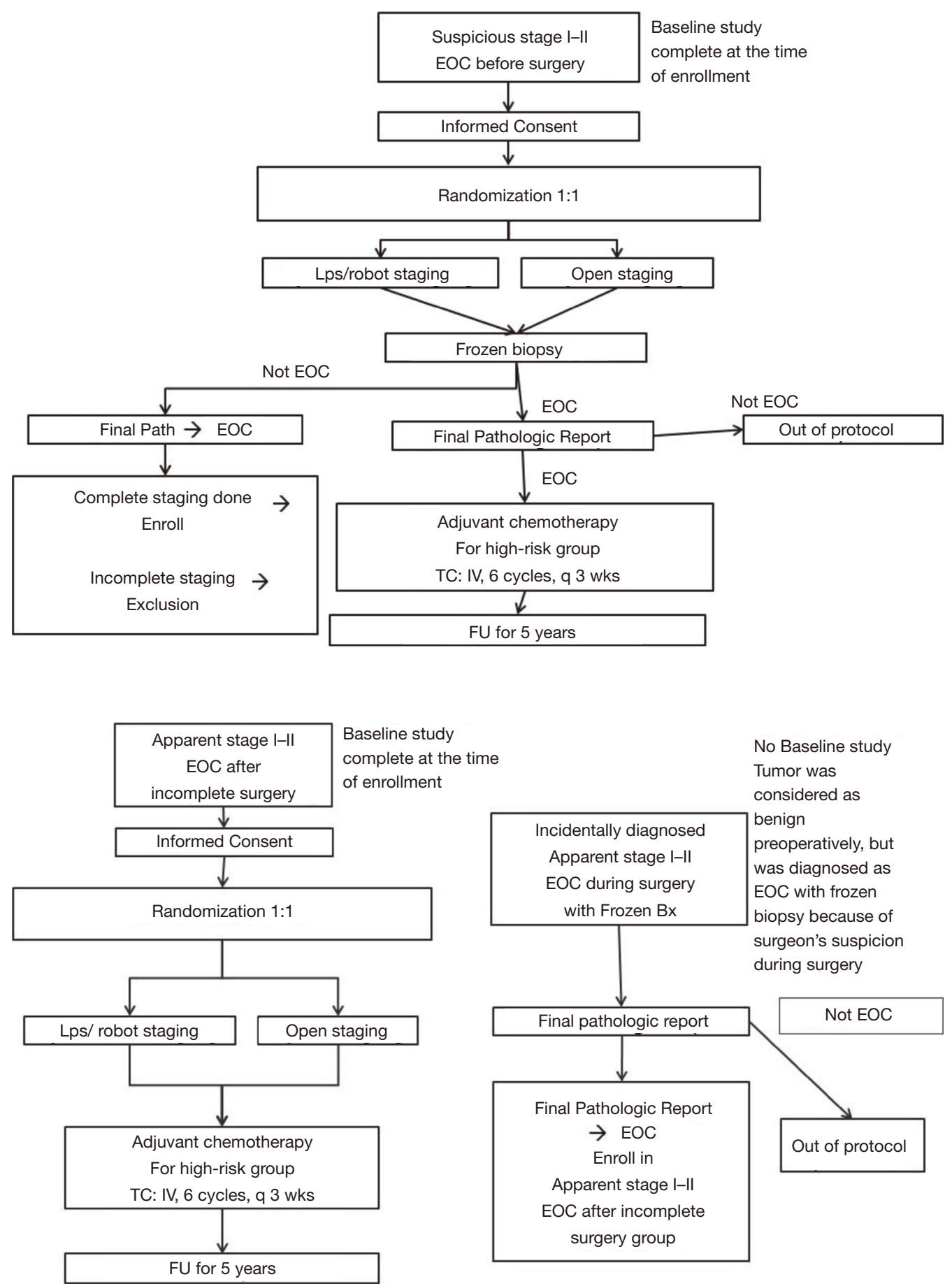

Figure 1 KGOG trials comparing surgical and survival outcomes between laparoscopic/robotic and open staging surgery in early-stage epithelial ovarian cancer. 
would require adequate selection and counseling of patients as well as careful selection of the surgical technique.

\section{Acknowledgments}

Funding: None.

\section{Footnote}

Provenance and Peer Review: This article was commissioned by the Guest Editors (Sang Yoon Park, Jae Weon Kim) for the series "Ultra-Radical Surgery in Ovarian Cancer: Surgical Techniques for Gynecologic Oncologist" published in Gland Surgery. The article was sent for external peer review organized by the Guest Editors and the editorial office.

Conflicts of Interest: All authors have completed the ICMJE uniform disclosure form (available at http://dx.doi. org/10.21037/gs-2019-ursoc-07). The series "UltraRadical Surgery in Ovarian Cancer: Surgical Techniques for Gynecologic Oncologist" was commissioned by the editorial office without any funding or sponsorship. The authors have no other conflicts of interest to declare.

Ethical statement: The authors are accountable for all aspects of the work in ensuring that questions related to the accuracy or integrity of any part of the work are appropriately investigated and resolved.

Open Access Statement: This is an Open Access article distributed in accordance with the Creative Commons Attribution-NonCommercial-NoDerivs 4.0 International License (CC BY-NC-ND 4.0), which permits the noncommercial replication and distribution of the article with the strict proviso that no changes or edits are made and the original work is properly cited (including links to both the formal publication through the relevant DOI and the license). See: https://creativecommons.org/licenses/by-nc-nd/4.0/.

\section{References}

1. Holschneider CH, Berek JS. Ovarian cancer: epidemiology, biology, and prognostic factors. Semin Surg Oncol 2000;19:3-10.

2. Slotman BJ, Rao BR. Ovarian cancer (review). Etiology, diagnosis, prognosis, surgery, radiotherapy, chemotherapy and endocrine therapy. Anticancer Res 1988;8:417-34.
3. Benedet JL, Bender H, Jones H, 3rd, et al. FIGO staging classifications and clinical practice guidelines in the management of gynecologic cancers. Int J Gynaecol Obstet 2000;70:209-62.

4. Petterson F. Annual report on the results of treatment in gynecological cancer. Twenty-first volume statements of results obtained in patients 1982 to 1986, inclusive 3and 5-year survival up to 1990. Int J Gynaecol Obstet 1991;36:1-315.

5. Jemal A, Siegel R, Ward E, et al. Cancer statistics, 2006. CA Cancer J Clin 2006;56:106-30.

6. Jemal A, Siegel R, Xu J, et al. Cancer statistics, 2010. CA Cancer J Clin 2010;60:277-300.

7. Monga M, Carmichael JA, Shelley WE, et al. Surgery without adjuvant chemotherapy for early epithelial ovarian carcinoma after comprehensive surgical staging. Gynecol Oncol 1991;43:195-7.

8. Young RC, Walton LA, Ellenberg SS, et al. Adjuvant therapy in stage I and stage II epithelial ovarian cancer. Results of two prospective randomized trials. N Engl J Med 1990;322:1021-7.

9. Querleu D, LeBlanc E. Laparoscopic infrarenal paraaortic lymph node dissection for restaging of carcinoma of the ovary or fallopian tube. Cancer 1994;73:1467-71.

10. Pomel C, Provencher D, Dauplat J, et al. Laparoscopic staging of early ovarian cancer. Gynecol Oncol 1995;58:301-6.

11. Amara DP, Nezhat C, Teng NN, et al. Operative laparoscopy in the management of ovarian cancer. Surg Laparosc Endosc 1996;6:38-45.

12. Leblanc E, Querleu D, Narducci F, et al. Laparoscopic restaging of early stage invasive adnexal tumors: a 10-year experience. Gynecol Oncol 2004;94:624-9.

13. Spirtos NM, Eisekop SM, Boike G, et al. Laparoscopic staging in patients with incompletely staged cancers of the uterus, ovary, fallopian tube, and primary peritoneum: a Gynecologic Oncology Group (GOG) study. Am J Obstet Gynecol 2005;193:1645-9.

14. Gallotta V, Petrillo M, Conte C, et al. Laparoscopic versus laparotomic surgical staging for early-stage ovarian cancer: a case-control study. J Minim Invasive Gynecol 2016;23:769-74.

15. Childers JM, Lang J, Surwit EA, et al. Laparoscopic surgical staging of ovarian cancer. Gynecol Oncol 1995;59:25-33.

16. Lee CL, Kay N, Chen HL, et al. The roles of laparoscopy in treating ovarian cancer. Taiwan J Obstet Gynecol 2009;48:9-14 
17. Ghezzi F, Malzoni M, Vizza E, et al. Laparoscopic staging of early ovarian cancer: results of a multi-institutional cohort study. Ann Surg Oncol 2012;19:1589-94.

18. Lee M, Kim SW, Paek J, et al. Comparisons of surgical outcomes, complications, and costs between laparotomy and laparoscopy in early-stage ovarian cancer. Int J Gynecol Cancer 2011;21:251-6.

19. Nezhat FR, Ezzati M, Chuang L, et al. Laparoscopic management of early ovarian and fallopian tube cancers: surgical and survival outcome. Am J Obstet Gynecol 2009;200:83.e1-6.

20. Chi DS, Abu-Rustum NR, Sonoda Y, et al. The safety and efficacy of laparoscopic surgical staging of apparent stage I ovarian and fallopian tube cancers. Am J Obstet Gynecol 2005;192:1614-9.

21. Ghezzi F, Cromi A, Uccella S, et al. Laparoscopy versus laparotomy for the surgical management of apparent early stage ovarian cancer. Gynecol Oncol 2007;105:409-13.

22. Park JY, Bae J, Lim MC, et al. Laparoscopic and laparotomic staging in stage I epithelial ovarian cancer: a comparison of feasibility and safety. Int J Gynecol Cancer 2008;18:1202-9.

23. Park JY, Kim DY, Suh DS, et al. Comparison of laparoscopy and laparotomy in surgical staging of earlystage ovarian and fallopian tubal cancer. Ann Surg Oncol 2008;15:2012-9.

24. Koo YJ, Kim JE, Kim YH, et al. Comparison of laparoscopy and laparotomy for the management of earlystage ovarian cancer: surgical and oncological outcomes. J Gynecol Oncol 2014;25:111-7.

25. Lu Q, Qu H, Liu C, et al. Comparison of Laparoscopy and Laparotomy in Surgical Staging of Apparent Early Ovarian Cancer: 13-year Experience. Medicine (Baltimore) 2016;95:e3655

26. Chereau E, Lavoue V, Ballester M, et al. External validation of a laparoscopic-based score to evaluate resectability for patients with advanced ovarian cancer undergoing interval debulking surgery. Anticancer Res 2011;31:4469-74.

27. Wu TI, Lee CL, Liao PJ, et al. Survival impact of initial surgical approach in stage I ovarian cancer. Chang Gung Med J 2010;33:558-67.

Cite this article as: Lim CK, Kim DY, Cho A, Choi JY, Park JY, Kim YM. Role of minimally invasive surgery in early ovarian cancer. Gland Surg 2021;10(3):1252-1259. doi: 10.21037/gs-2019ursoc-07
28. Vergote I. Prognostic factors in stage I ovarian carcinoma. Verh K Acad Geneeskd Belg 2001;63:257-71; discussion 272-6.

29. Zivanovic O, Sonoda Y, Diaz JP, et al. The rate of portsite metastases after 2251 laparoscopic procedures in women with underlying malignant disease. Gynecol Oncol 2008;111:431-7.

30. Ramirez PT, Wolf JK, Levenback C. Laparoscopic portsite metastases: etiology and prevention. Gynecol Oncol 2003;91:179-89.

31. Panici PB, Palaia I, Bellati F, et al. Laparoscopy compared with laparoscopically guided minilaparotomy for large adnexal masses: a randomized controlled trial. Obstet Gynecol 2007;110:241-8.

32. Ding X, Zhang T, Hou Q, et al. A contrast analysis of roboticassisted gynecological surgery, laparoscopy and open abdomen operation in the treatment of cervical cancer. Chin Clin Oncol 2014;19:608-12.

33. Feuer GA, Lakhi N, Barker J, et al. Perioperative and clinical outcomes in the management of epithelial ovarian cancer using a robotic or abdominal approach. Gynecol Oncol 2013;131:520-4.

34. Magrina JF, Zanagnolo V, Noble BN, et al. Robotic approach for ovarian cancer: perioperative and survival results and comparison with laparoscopy and laparotomy. Gynecol Oncol 2011;121:100-5.

35. Romagnolo C, Gadducci A, Sartori E, et al. Management of borderline ovarian tumors: results of an Italian multicenter study. Gynecol Oncol 2006;101:255-60.

36. Schilling MK, Redaelli C, Krahenbuhl L, et al. Splanchnic microcirculatory changes during CO2 laparoscopy. J Am Coll Surg 1997;184:378-82.

37. Targarona EM, Martinez J, Nadal A, et al. Cancer dissemination during laparoscopic surgery: tubes, gas, and cells. World J Surg 1998;22:55-60.

38. Allardyce RA, Morreau P, Bagshaw PF. Operative factors affecting tumor cell distribution following laparoscopic colectomy in a porcine model. Dis Colon Rectum 1997;40:939-45.

39. Ramirez PT, Frumovitz M, Pareja R, et al. Minimally Invasive versus Abdominal Radical Hysterectomy for Cervical Cancer. N Engl J Med 2018;379:1895-904. 